Case Reort:

\title{
Hydroxychloroquine-induced Unusual Generalized Pustular Cutaneous Reaction As a New Clinical En- tity: A Case Series
}

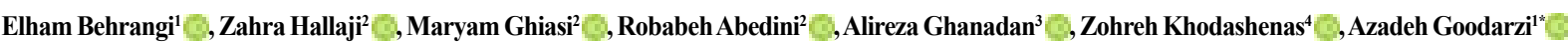

1. Department of Dermatology, Rasoul Akram Hospital, Iran University of Medical Sciences, Tehran, Iran

2. Department of Dermatology, Razi Hospital, Tehran University of Medical Sciences, Tehran, Iran.

3. Department of Dermatopathology, Razi Hospital, Tehran University of Medical Sciences, Tehran, Iran.

4. Department of Dermatology, Faculty of Medicine, Shahed University of Medical Sciences, Tehran, Iran.

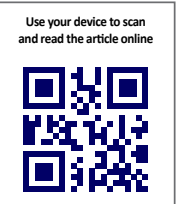

Crtation Behrangi E, Hallaji Z, Ghiasi M, Abedini R, Ghanadan A, Khodashenas Z, Goodarzi A. Hydroxychloroquine-induced Unusual Generalized Pustular Cutaneous Reaction As a New Clinical Entity: A Case Series. Immunoregulation. 2020; 3(1):67-72. http://dx.doi.org/10.32598/Immunoregulation.3.1.8

http://dx.doi.org/10.32598/Immunoregulation.3.1.8

Article info:

Received: 03 Mar 2020

Accepted: 06 May 2020

Available Online: 01 Jul 2020

Keywords:

Hydroxychloroquine (HCQ), Pustular eruption, Pustular Psoriasis (PP), Acute Generalized Exanthematous Pustulosis (AGEP)

\begin{abstract}
A B S T R A C T
Background: Pustular Psoriasis (PP) and Acute Generalized Exanthematous Pustulosis (AGEP) clinically resemble each other. There are many patients with generalized pustular presentations like hydroxychloroquine-induced pustular reaction who do not completely fall within PP or AGEP categories and needed to be more evaluated. So we examined a large series of these reactions to hydroxychloroquine (HCQ) and reviewed the literature in this regard.

Materials and Methods: Records of 20 patients admitted to Razi Hospital, Tehran, Iran with acute onset of generalized pustular eruption following hydroxychloroquine therapy were retrieved, and their demographic and clinical features were reviewed. In the meantime, all related articles in PubMed and Google scholar databases were studied as well.

Results: All 20 patients were female with an Mean \pm SD age of $48.35 \pm 13.76$ years. The Mean \pm SD between the first dose and skin eruption was $3.62 \pm 1.42$ weeks. HCQ was prescribed for them, mainly for rheumatologic conditions and joint problems other than psoriasis. Treatment was planned according to the first clinical judgment, pathological findings, and clinical behavior of the disease. Eleven patients were treated as PP and 9 as AGEP.

Conclusion: No evident history of psoriasis, drug-related eruption, as well as rapid and complete response to steroids in some patients, favor AGEP diagnosis. However, the long interval of symptoms appearance, prolonged disease course, relapses despite discontinuation of the responsible drug, and no appropriate response to the steroid, favor PP. So, it could be a new entity.
\end{abstract}

* Corresponding Author:

Azadeh Goodarzi, PhD.

Address: Department of Dermatology, Rasoul Akram Hospital, Iran University of Medical Sciences, Tehran, Iran.

Phone: +98 (912) 882448

E-mail: goodarzi.a@iums.ac.ir 


\section{Introduction}

eneralized (von Zumbush) form of pustular psoriasis (PP) presents with an acute generalized pustular eruption on an edematous and erythematous skin and a high fever. The treatment needs systemic anti-psoriatic agents, sometimes in conjunction with periodical steroids administration. The disease tends to relapse, too. $\mathrm{PP}$ can occur in persons with prior psoriasis or de novo. Pregnancy, drugs, rapid tapering of steroids, and systemic infections are known as triggers of generalized pustular psoriasis although drugs are not common triggers $[1,2]$.

Acute Generalized Exanthematous Pustulosis (AGEP) has similar clinical features, commonly begins from the face and intertriginous areas. Approximately, 90\% of AGEPs are drug-induced, which occurs 4 days after consuming the responsible drug. AGEP improves within 1-2 weeks spontaneously with discontinuation of the responsible drug and supportive care or with a short course of topical or systemic steroids without any relapse unless after re-exposure [1, 3-10].

Hydroxychloroquine (HCQ) is an antimalarial drug used in different fields of medicine, especially in dermatology. HCQ is considered as an inducer or aggravator of plaquetype psoriasis, so it is not usually intended in the case of $\mathrm{PP}$ without any plaque-type precursor and there are only a few reports in this regard [1, 11-14]. However, HCQ is one of the causative drugs of AGEP - in some studies as a rare cause and in recent studies as a common cause $[1,15]$.

There are many reports of unusual AGEPs after usage of HCQ, mainly in the setting of rheumatologic problems, which are somehow similar to PP clinical course and characteristics [16-19]. These types of HCQ-induced AGEPs usually have longer intervals from drug initiation, as well as a more severe, prolonged, and recalcitrant course [20-35]. Also, there are many reports of Stevens-Johnson/toxic epidermal necrolysis or drug reaction with eosinophilia and systemic symptoms-like AGEPs, especially as HCQ-induced AGEP [36-40].

Concerning clinical similarities of these 2 entities, history, physical exam, clinical course, and response to treatment as well as histopathology can help us in the differential diagnosis. For example, the overall history of recent drug usage is in favor of AGEP rather than PP; severe edema of superficial dermis, necrosis of keratinocytes, and exocytosis of eosinophils in pathology are more in favor of AGEP, whereas acanthosis more in favor of PP $[1,41-43]$.
Although there are many reports of HCQ-induced AGEP or PP (that in some cases are atypical or overlapping between two entities), this study proposes the overlap presentation as a new entity and explains it by detail based on the largest series of similar cases. Based on the observation of patients with pustular reactions to HCQ (hospitalized in Razi Hospital), their characteristics were not completely consistent with AGEP or PP. Also, there were many problems and complications in their diagnosis and treatment. Thus, we designed a comprehensive large case series study about different clinical and therapeutic features of these patients also review the literature in this regard.

\section{Materials and Methods}

We investigated records of patients hospitalized at skin specialized Razi Hospital, Tehran, Iran that discharged with a diagnosis of drug reaction AGEP and PP over 3.5 years in a cross-sectional study. Finally, we found 20 cases of generalized pustular skin eruption following the taking of HCQ, and the admission records and follow up records of these patients were used for this large case series. The final assessment of the clinical manifestations, disease course, and treatment protocol had been performed $\mathrm{f}$ tor all these 20 cases. After discharge of the patients, we designed an interpersonal pathological overview of available samples and determined one pathologic diagnosis for each sample based on the most votes, retrospectively. We discussed the most probable clinical and histopathological diagnosis for each patient and disease course and management of the disorder. All related articles in PubMed and Google scholar databases were reviewed and discussed in this regard, too.

\section{Results}

\section{Demographic data}

All 20 cases were female and their average \pm SD age was $48.35 \pm 13.76$ years (ranged 14-69 years). The Mean \pm SD interval between the onset of drug usage and the initiation of the cutaneous pustular reaction was $3.62 \pm 1.42$ weeks (ranged 2-7 weeks).

HCQ was administered for rheumatologic diseases and joint problems (such as RA) in $85 \%$ of cases. Except for one patient with a history of skin lesions of psoriasis and joint pain related to psoriatic arthritis, other patients had no history of psoriatic skin or joint problems or triggering factors for PP.

All patients had mild to moderate fever at the time of admission and had a slight ESR increase and leukocy- 
Table 1. The first diagnosis and final interpersonal diagnosis, as well as the final successful therapeutic protocol

\begin{tabular}{|c|c|c|c|c|c|}
\hline \multicolumn{6}{|c|}{ Pustular Reaction to HCQ Clinically } \\
\hline $\begin{array}{l}\text { The Diagnosis Which Was Recorded in Patients' File } \\
\text { Based on Primary Histopathologic Evaluation }\end{array}$ & AGEP & PP & AGEP-PP Overlap & $\begin{array}{l}\text { Lichenoid Drug } \\
\text { Reaction }\end{array}$ & PSO \\
\hline $\begin{array}{l}\text { Available sample for histopathologic interpersonal } \\
\text { overview in this study }\end{array}$ & 8 & 2 & 4 & 1 & 1 \\
\hline $\begin{array}{l}\text { The final diagnosis of histopathologic overview } \\
\text { in this study }\end{array}$ & $\begin{array}{c}\text { 4PP } \\
3 P S O \\
1 \text { AGEP }\end{array}$ & $2 \mathrm{PP}$ & $\begin{array}{l}\text { 2PP } \\
\text { 2AGEP }\end{array}$ & 1AGEP & 1AGEP \\
\hline $\begin{array}{l}\text { Final therapeutic management during hospitalization of } \\
\text { patients }\end{array}$ & $\begin{array}{l}\text { 6AGEP } \\
2 \mathrm{PP}\end{array}$ & $2 \mathrm{PP}$ & 4PP & 1AGEP & 1AGEP \\
\hline
\end{tabular}

HCQ: Hydroxychloroquine; PP: Pustular Psoriasis; AGEP: Acute Generalized Exanthematous Pustulosis; PSO: Psoriasis

tosis in some patients. Their Mean \pm SD hospitalization time was 11.50 \pm 7.14 days (ranged 3-29 days ).

Seventy percent of the patients were biopsied at least 2 times during hospitalization for exact clinical diagnosis and appropriate treatment plan. (Disease clinic and course was not completely compatible with AGEP or PP, which necessitated more than one biopsy in the majority of patients).

All patients received topical steroids as part of their treatments. Four patients in the primary histopathologic assessment were diagnosed as AGEP / PP overlap, being treated based on clinical judgment of dermatologist, as PP in the primary treatment plan. Of 9 patients with histopathologic diagnosis of AGEP, 7 patients were treated with systemic steroids with the Mean \pm SD dose of $26.14 \pm 1.20 \mathrm{mg} / \mathrm{d}$ (1 patient treated with only topical steroids and 1 patient as PP) in the primary treatment plan. Of 3 patients with histopathologic diagnosis of $\mathrm{PP}$, all managed as PP. Of 4 patients with pathologic report as the drug reaction, 1 patient with histopathologic evidence of psoriasis was managed in the primary treatment plan as PP and the remaining patients as AGEP.

\section{Course and management data}

Finally, as an ultimate treatment protocol plan in the period of admission, 11 patients out of 20 total cases managed as PP and 9 patients as AGEP. You can see Table 1 for more information. The Mean \pm SD age of the patients managed as PP was $52.72 \pm 11.24$ years. The Mean \pm SD duration of admission was $14 \pm 8.5$ days and the time interval between drug initiation and the reaction was $4.22 \pm 1.50$ weeks. The Mean $\pm \mathrm{SD}$ age of the patients managed as AGEP was $43 \pm 15.27$ years, and the Mean \pm SD duration of admission was $8.44 \pm 3.3$ days. The Mean \pm SD time interval between drug initiation and the reaction was $2.88 \pm 0.92$ weeks.
These three variables (age, duration of admission, and interval of reaction) were significantly different between finally diagnosed AGEP or PP ( $\mathrm{P}=0.00$ for all of them). Two patients who managed as $\mathrm{PP}$, despite appropriate treatment, re-hospitalized due to flare of disease during the first month of discharge, and some patients in this group, experienced crops of minor flares during hospitalization.

\section{Discussion}

In de novo or triggered generalized pustular psoriasis, the major symptom of the patient is fever and illness accompanies pustular reaction. In the setting of triggering factors like pregnancy, drug, or hypocalcemia, the clinical outcome may be more favorable but in de novo cases, we usually expect recalcitrant and recurrent chronic course with exacerbated and remitted phases. In the case of drug-induced pustular psoriasis, a few weeks interval period is usually expected for the evident cutaneous presentation. In AGEP, we usually encounter a febrile and ill patient who is referred a few days after consumption of responsible drug and favorably responses to therapy without any relapse in drugfree periods. However, there are many patients with generalized pustular reaction, especially after HCQ use, who present with incomplete clinical and histopathologic criteria of AGEP or PP [1-10].

Although HCQ is one of the well-known drugs related to drug-induced or drug-aggravated AGEP or PP, in many cases of HCQ-induced pustular cutaneous eruptions, clinical and histopathologic criteria cannot be fully interpreted as AGEP or PP. Due to the complexity of clinical and histopathological differences between AGEP and PP in pustular reactions induced by HCQ and also difficulties in dealing with how to manage such reactions, we decided to conduct a case series study to further evaluate this clinicopathological dilemma in dermatology. 
AGEP is considered as a drug-induced condition with two peaks of skin eruption, 1 day for antibiotics, 11 days for other drugs such as HCQ, and an average of less than 96 hours $[1,7]$. In our study, however, the Mean \pm SD of time interval between drug consumption and the reaction was $3.62 \pm 1.42$ weeks ( 25 days) which is about 2 times more than the maximum interval in AGEP. So the onset is not consistent with AGEP; however, $45 \%$ of the patients were managed as AGEP without any relapse. We can explain this long interval with probable PP; considering that the patients did not have a history of any psoriasis except one, so drug-induced de novo PP sounds a good diagnosis, as $55 \%$ of the patients were managed as PP and some experienced many courses of relapse during therapy or tapering of therapy like PP. It is supposed that the rheumatologic problems of the patients might be non-diagnosed psoriatic arthritis without any cutaneous manifestation.

Recently, biallelic mutations in the gene encoding IL36 receptor antagonist - which itself leads to increased IL8 and response to IL1B - have been identified in PP. Also, HLAB5, DR11, and DQ3 have been proposed for AGEP [1]. The genetic and immunologic susceptibility to HCQinduced pustular cutaneous reaction may play an important role in this process or HCQ may induce a unique type of cutaneous reaction presented as AGEP-PP overlap. So regarding drug-induced nature, it is really in favor of AGEP, and regarding interval, it is really in favor of PP and about its course and management, it may be consistent with AGEP or PP (45\% and 55\%, respectively) [20-35].

Pustular reaction due to HCQ will be in favor of AGEP if these conditions are present: no evident history of psoriasis, no pustular eruption after recent consumption of a new drug, rapid and complete response to systemic steroid and even management of 1 case only with a topical steroid, as well as no relapse in at least $45 \%$ of the patients. On the other hand, the diagnosis favors PP when these conditions are present: the long interval between drug initiation and reaction and the duration of the disease, some periods of relapses despite discontinuation of the responsible drug at admission time and after discharge, also no appropriate response to systemic steroid as a mainstay of treatment in $55 \%$ of the cases.

There are still some questions here. Regarding sex predilection and the dominant history of the rheumatologic disorder, is it logical to consider hormonal or genetic predispositions? Because most patients were managed as PP, is it possible that the joint disorders be the first signs of psoriasis before the onset of other psoriatic signs and symptoms? Because the average age, the average interval between the drug initiation and reaction and duration of admission were significantly different between the patients managed as AGEP or PP, is it possible that HCQ acts as both inducers of AGEP or de novo PP based on the genetic, immunologic, and hormonal susceptibilities?

Besides the well-known pustular reaction to HCQ in adults and even in children [44], it may be necessary to define a hydroxychloroquine-induced pustular reaction which is not completely consistent with AGEP or PP - as a new entity in dermatology. It is a long way to do new research with these hypotheses.

We did not mention the primary location of lesions, their spread, and distribution and mucosal involvement because of the shortages of recorded data. The follow-up records were not complete and the outcomes of these patients were not available. However, 6 months follow-up of 8 patients whose data were available revealed good response to therapy and slow tapering of treatment, without any relapse. Despite no history of psoriasis and the first diagnosis of AGEP, one patient showed cutaneous lesions compatible with plaque-type psoriasis over time. One of the explanations for missing follow-up could be the appropriate response to therapy without any relapse. In most cases, the definite diagnosis of the joint problem was not clear to discuss more.

\section{Ethical Considerations}

\section{Compliance with ethical guidelines}

The study was performed in compliance with the guidelines of the Medical Ethics Committee of Iran University of Medical Sciences.

\section{Funding}

This research did not receive any specific grant from funding agencies in the public, commercial, or non-profit sectors.

\section{Authors' contributions}

Investigation, writing the original draft, concept, design, critical revision of the manuscript: Zahra Hallaji, Azadeh Goodarzi, Elham Behrangi; Data collection: Azadeh Goodarzi; Review, investigation, data analysis, read and approving the final manuscript: All authors.

\section{Conflicts of interest}

The authors declared no conflicts of interest. 


\section{Acknowledgements}

The authors would like to thank the Rasoul Akram Hospital Clinical Research Development Center (RCRDC) for technical and editorial assistance.

\section{References}

[1] Goodman WT, Barrett TL, AGEP, Bolognia JL, Jorrizo Jl, Schafter JV. Dermatology. Philadelphia: Elsevier Saunders; 2012.

[2] de Sousa AS, de Castro Lara OAC, Papaiordanou F, Simioni Marchioro GS, Tebcherani AJ. Acute generalized exanthematous pustulosis $x$ Von Zumbusch's pustular psoriasis: A diagnostic challenge in a psoriatic patient. Anais Brasileiros de Dermatologia. 2015; 90(4):557-60. [DOI:10.1590/abd18064841.20153256] [PMID] [PMCID]

[3] De A, Das S, Sarda A, Pal D, Biswas P. Acute generalised exanthematous pustulosis: An update. Indian Journal of Dermatology. 2018; 63(1):22-9. [DOI:10.4103/ijd.IJD_581_17] [PMID] [PMCID]

[4] Fernando SL. Acute generalised exanthematous pustulosis. Australasian Journal of Dermatology. 2012; 53(2):87-92. [DOI:10.1111/j.1440-0960.2011.00845.x] [PMID]

[5] Lee HY, Chou D, Pang SM, Thirumoorthy T. Acute generalized exanthematous pustulosis: Analysis of cases managed in a tertiary hospital in Singapore. International Journal of Dermatology. 2010; 49(5):507-12. [DOI:10.1111/j.13654632.2010.04313.x] [PMID]

[6] Mebazaa A, Kort R, Zaiem A, Elleuch D, Moula H, Cheikhrouhou $\mathrm{R}$, et al. [Acute generalized exanthematous pustulosis. Study of 22 cases (French)]. La Tunisie Médicale. 2010; 88(12):910-5. [PMID]

[7] Sidoroff A, Dunant A, Viboud C, Halevy S, Bouwes Bavinck JN, Naldi L, et al. Risk factors for Acute Generalized Exanthematous Pustulosis (AGEP)-results of a multinational case-control study (EuroSCAR). British Journal of Dermatology. 2007; 157(5):989-96. [DOI:10.1111/j.1365-2133.2007.08156.x] [PMID]

[8] Speeckaert MM, Speeckaert R, Lambert J, Brochez L. Acute generalized exanthematous pustulosis: An overview of the clinical, immunological and diagnostic concepts. European Journal of Dermatology. 2010; 20(4):425-33. [DOI:10.1684/ ejd.2010.0932] [PMID]

[9] Szatkowski J, Schwartz RA. Acute Generalized Exanthematous Pustulosis (AGEP): A review and update. Journal of the American Academy of Dermatology. 2015; 73(5):843-8. [DOI:10.1016/j.jaad.2015.07.017] [PMID]

[10] Feldmeyer L, Heidemeyer K, Yawalkar N. Acute Generalized Exanthematous Pustulosis: Pathogenesis, genetic background, clinical variants and therapy. International Journal of Molecular Sciences. 2016; 17(8):1214. [DOI:10.3390/ ijms17081214] [PMID] [PMCID]

[11] Friedman SJ. Pustular psoriasis associated with hydroxychloroquine. Journal of the American Academy of Dermatology. 1987; 16(6):1256-7. [DOI:10.1016/S0190-9622(87)80021-X] [PMID]
[12] Vine JE, Hymes SR, Warner NB, Cohen PR. Pustular psoriasis induced by hydroxychloroquine: $\mathrm{A}$ case report and review of the literature. The Journal of Dermatology. 1996; 23(5):357-61. [PMID]

[13] Degos R, Touraine R, Civatte J, Belauich S. [Erythroderma with appearance of pustular psoriasis during a case of Duhring's disease treated with hydroxychloroquine (French)]. Bulletin de la Société Française de Dermatologie et de Syphiligraphie. 1963; 70:129-34. [PMID]

[14] Maglie R, Dini V, Romanelli M. Pustular psoriasis induced by hydroxychloroquine: A case report to confirm a rare association. Journal of the American Academy of Dermatology. 2016; 74(5 Suppl 1):AB265. [DOI:10.1016/j. jaad.2016.02.1027.]

[15] Saissi EH, Beau-Salinas F, Jonville-Béra AP, Lorette G, Autret-Leca E, de Pharmacovigilance CR. [Drugs associated with acute generalized exanthematic pustulosis (French)] Annales de Dermatologie et de Vénéréologie. 2003; 130(67):612-8. [PMID]

[16] Benjegerdes KE, Hyde K, Kivelevitch D, Mansouri B. Pustular psoriasis: Pathophysiology and current treatment perspectives. Psoriasis (Auckland, N.Z.). 2016; 6:131-44. [DOI:10.2147/PTT.S98954] [PMID] [PMCID]

[17] Choon SE, Lai NM, Mohammad NA, Nanu NM, Tey KE, Chew SF. Clinical profile, morbidity, and outcome of adultonset generalized pustular psoriasis: Analysis of 102 cases seen in a tertiary hospital in Johor, Malaysia. International Journal of Dermatology. 2014; 53(6):676-84. [DOI:10.1111/ ijd.12070] [PMID]

[18] Hoegler KM, John AM, Handler MZ, Schwartz RA. Generalized pustular psoriasis: A review and update on treatment. Journal of the European Academy of Dermatology and Venereology. 2018; 32(10):1645-51. [DOI:10.1111/jdv.14949] [PMID]

[19] Weisenseel P, Wilsmann-Theis D, Kahl C, Reich K, Mössner R. [Pustular psoriasis (German)]. Der Hautarzt. 2016; 67(6):445-53. [DOI:10.1007/s00105-016-3804-4] [PMID]

[20] Evans CC, Bergstresser PR. Acute generalized exanthematous pustulosis precipitated by hydroxychloroquine. Journal of the American Academy of Dermatology. 2004; 50(4):6501. [DOI:10.1016/S0190-9622(03)02733-6]

[21] Fain O, Stirnemann J, Kettaneh A. [Acute generalized exanthematous pustulosis included by hydroxychloroquine during lupus (French)]. La Revue du Praticien. 2010; 60(1):20. [PMID]

[22] Martins A, Lopes LC, Paiva Lopes MJ, Rodrigues JC. Acute generalized exanthematous pustulosis induced by hydroxychloroquine. European Journal of Dermatology. 2006; 16(3):317-8. [PMID]

[23] Assier-Bonnet H, Saada V, Bernier M, Clerici T, Saïag P. Acute generalized exanthematous pustulosis induced by hydroxychloroquine. Dermatology. 1996; 193(1):70-1. [DOI:10.1159/000246211] [PMID]

[24] Bailey K, McKee D, Wismer J, Shear N. Acute generalized exanthematous pustulosis induced by hydroxychloroquine: First case report in Canada and review of the literature. Journal of Cutaneous Medicine and Surgery. 2013; 17(6):414-8. [DOI:10.2310/7750.2013.12105] [PMID]

[25] Charfi O, Kastalli S, Sahnoun R, Lakhoua Gh. Hydroxychloroquine-induced acute generalized exanthematous pustulo- 
sis with positive patch-testing. Indian Journal of Pharmacology. 2015; 47(6):693-4. [DOI:10.4103/0253-7613.169589] [PMID] [PMCID]

[26] Djennane M, Tablit I, Billhot M, Banal F. [Acute generalized exanthematic pustulosis induced by hydroxychloroquine: Case report (French)]. La Revue de Médecine Interne. 2010; 31(3):e7-e8. [DOI:10.1016/j.revmed.2009.03.350] [PMID]

[27] Duman H, Topal IO, Kocaturk E, Cure K, Mansuroglu I. Acute generalized exanthematous pustulosis induced by hydroxychloroquine: A case with atypical clinical presentation. Anais Brasileiros de Dermatologia. 2017; 92(3):404-6. [DOI:10.1590/abd1806-4841.20175561] [PMID] [PMCID]

[28] Lotem M, Ingber A, Segal R, Sandbank M. Generalized pustular drug rash induced by hydroxychloroquine. Acta Dermato-Venereologica. 1990; 70(3):250-1. [PMID]

[29] Paradisi A, Bugatti L, Sisto T, Filosa G, Amerio PL, Capizzi R. Acute generalized exanthematous pustulosis induced by hydroxychloroquine: Three cases and a review of the literature. Clinical Therapeutics. 2008; 30(5):930-40. [DOI:10.1016/j.clinthera.2008.05.014] [PMID]

[30] Park JJ, Yun SJ, Lee JB, Kim SJ, Won YH, Lee SC. A case of hydroxychloroquine induced acute generalized exanthematous pustulosis confirmed by accidental oral provocation. Annals of Dermatology. 2010; 22(1):102-5. [DOI:10.5021/ ad.2010.22.1.102] [PMID] [PMCID]

[31] Pastushenko I, Gracia-Cazaña T, Morales-Moya AL, Pilar Grasa M. [Acute cutaneous pustular eruption due to hydroxychloroquine (Spanish)]. Medicina Clínica. 2014; 143(7):e13. [DOI:10.1016/j.medcli.2014.04.003] [PMID]

[32] Mohaghegh F, Jelvan M, Rajabi P. A case of prolonged generalized exanthematous pustulosis caused by hydroxychloroquine-Literature review. Clinical Case Reports. 2018; 6(12):2391-5. [DOI:10.1002/ccr3.1811] [PMID] [PMCID]

[33] Pearson KC, Morrell DS, Runge SR, Jolly P. Prolonged pustular eruption from hydroxychloroquine: An unusual case of acute generalized exanthematous pustulosis. Cutis. 2016; 97(3):212-6. [PMID]

[34] Castner NB, Harris JC, Motaparthi K. Cyclosporine for corticosteroid-refractory acute generalized exanthematous pustulosis due to hydroxychloroquine. Dermatologic Therapy. 2018; 31(5):e12660. [DOI:10.1111/dth.12660] [PMID]

[35] Yalçın B, Çakmak S, Yıldırım B. Successful treatment of hydroxychloroquine-induced recalcitrant acute generalized exanthematous pustulosis with cyclosporine: Case report and literature review. Annals of Dermatology. 2015; 27(4):431-4. [DOI:10.5021/ad.2015.27.4.431] [PMID] [PMCID]

[36] Kostopoulos TC, Krishna SM, Brinster NK, Ortega-Loayza AG. Acute generalized exanthematous pustulosis: Atypical presentations and outcomes. Journal of the European Academy of Dermatology and Venereology. 2015; 29(2):209-14. [DOI:10.1111/jdv.12721] [PMID]

[37] Lateef A, Tan KB, Lau TC. Acute generalized exanthematous pustulosis and toxic epidermal necrolysis induced by hydroxychloroquine. Clinical Rheumatology. 2009; 28(12):1449. [DOI:10.1007/s10067-009-1262-4] [PMID]

[38] Mercogliano C, Khan M, Lin C, Mohanty E, Zimmerman R. AGEP overlap induced by hydroxychloroquine: A case report and literature review. Journal of Community Hospital
Internal Medicine Perspectives. 2018; 8(6):360-2. [DOI:10.108 0/20009666.2018.1547089] [PMID] [PMCID]

[39] Peermohamed Sh, Haber RM. Acute generalized exanthematous pustulosis simulating toxic epidermal necrolysis: A case report and review of the literature. Archives of Dermatology. 2011; 147(6):697-701. [DOI:10.1001/archdermatol.2011.147] [PMID]

[40] Worsnop F, Chong H, Ostlere L, Natkunarajah J. Acute generalized exanthematous pustulosis mimicking toxic epidermal necrolysis in patients with psoriasis: A coincidence? Clinical and Experimental Dermatology. 2015; 40(6):688-9. [DOI:10.1111/ced.12590] [PMID]

[41] Kardaun SH, Kuiper H, Fidler V, Jonkman MF. The histopathological spectrum of Acute Generalized Exanthematous Pustulosis (AGEP) and its differentiation from generalized pustular psoriasis. Journal of Cutaneous Pathology. 2010; 37(12):1220-9. [DOI:10.1111/j.1600-0560.2010.01612.x] [PMID]

[42] Halevy S, Kardaun SH, Davidovici B, Wechsler J, Euro SCAR and Regi SCAR Study Group. The spectrum of histopathological features in acute generalized exanthematous pustulosis: A study of 102 cases. The British Journal of Dermatology. 2010; 163(6):1245-52. [DOI:10.1111/j.13652133.2010.09967.x] [PMID]

[43] Vassallo C, Derlino F, Brazzelli V, D'Ospina RD, Borroni G. Acute generalized exanthematous pustulosis: Report of five cases and systematic review of clinical and histopathological findings. Giornale Italiano di Dermatologia e Venereologia. 2014; 149(3):281-90. [PMID]

[44] Liccioli G, Marrani E, Giani T, Simonini G, Barni S, Mori F. The first pediatric case of acute generalized exanthematous pustulosis caused by hydroxychloroquine. Pharmacology. 2019; 104(1-2):57-9. [DOI:10.1159/000500406] [PMID] 